\title{
THE SEISMIC BEHAVIOR OF BURIED SEABED WALLS IN LIQUEFACTION SOIL
}

\author{
Javad Royaei and Kabir Sadeghi \\ Department of Civil and Environmental Engineering, Near East University, \\ Boulevard, Turkey; mahaniranian1@gmail.com
}

\begin{abstract}
The present study aimed to investigate the seismic behavior of enclosed seawater walls, the buried site of which lies in liquefaction soil. An experimental specimen was manufactured and tested on the seismic table, and a numerical study was also modeled in the ABAQUS software based on the experimental outcomes. In both the experimental and numerical studies, a susceptible liquefaction layer around the root of the wall was considered due to the root lean soil leakage and large lateral pressure, and the soil behind the root caused the failure of the buried section. According to the results, the lateral movement significantly decreased due to the backing effect of this layer on the buried section of the wall. Furthermore, an active wedge was formed from the buried side to the back of the containment, and the braces were overwhelming due to the presence of the locks in the wedge and their movement along with the wedge. The displacement of the crown and foot of the wall decreased with the increased base acceleration and higher frequency of the entrance movement.
\end{abstract}

\section{KEYWORDS}

Coastal wall, Liquefaction, Improvement, Seismic behavior

\section{INTRODUCTION}

Given the strategic role of coastal structures in the economy of every country and the geographical location of Iran in the earthquake zone, the seismic study of coastal walls is of paramount importance. The damages observed in recent earthquakes (e.g., 1995 Japan Kobe earthquake and 1999 Taiwan Chichi) indicate the great displacements of coastal walls, while further damages have also occurred due to the deformation of a soft layer or layers of soil/pier walls. In the cases where landfill and soil have not been leak-proof, the walls have been reported to exhibit safety behaviors during an earthquake [1]. As coastal soils and sea sediments, the density is often low to moderate, while there is a high likelihood of susceptible layers to liquefaction in soil. Therefore, the effects of these layers on the prediction of the seismic behavior of coastal walls must be properly examined.

Curtain walls are a common type of coastal walls, which are supported by the lateral pressure of soil and water through various components (e.g., shield, harness, and bar), as well as the inactive passive soil pressure in front of the buried wall. The static behavior of these structures is assessed based on the conventional estimation methods of soil lateral pressure in extreme states and establishing the equilibrium force and anchor ratios using a joint foot or the foot method [2]. However, the dynamic behavior of restored walls is more complicated, which is mainly due to the multiplicity of the influential factors in their behavior and influence. Restored walls are mutually supportive. In addition to the performance of the components, the response of these structures strongly affects the dynamic behavior of the soil relative to the input motion [3]. The seismic performance of these walls is defined based on their ability to operate and the amount of the damage caused by tension (e.g., displacement). The relevant parameters in this regard include the displacements of important areas (e.g., displacement of the shield and deck) and containment or the cracking of the surrounding soil, 
and the tensions of the component instruments, such as the shield tensions in the upper and buried areas, stresses of the inhibitory cable, and barrier stress.

In performance-based design, it is essential to define the injury criteria by selecting the appropriate factors. In addition, the desirable process of failure with increased earthquake load should be defined for restored walls. For instance, if shield repair is difficult, the containment must be surrendered before shield submission. Since it is often more difficult to repair the buried section of a wall, this section has a higher seismic resistance and should be avoided by breaking the shield, interlock cable, and rupture. In addition to the features of the instrument parts (shield, bar, and inhibition), the stability of these structures depends on the geotechnical conditions of the bedding and landing.

In general, three friction modes could be considered for these structures. If a failure occurs in an inhibition rod or shield, the failure of the set is classified as shift/shaft shaping/shift. The change in the location of a set is due to the deformation or rupture of the containment or the rupture of a set known as shape/break in restraint. If the soil around the buried length cannot prevent the wall from rising, the failure will be referred to as a 'bursting section failure'. According to the field studies in this regard, the presence of a liquefaction layer in sections of the soot or lower soil leads to significantly larger shape variations. In addition to the existing walls that have been constructed without any liquefaction hazard in susceptible liquefied soils, several cases of the appearance of such layers have been reported in the sector where there are sensitive coastal walls. Considering that walls often have a considerable length, inevitable changes in the type of the layers may lead to the placement of a lubricating layer in one or more structural parts of the structure. Figure 1 shows the possible states of liquefaction layering.

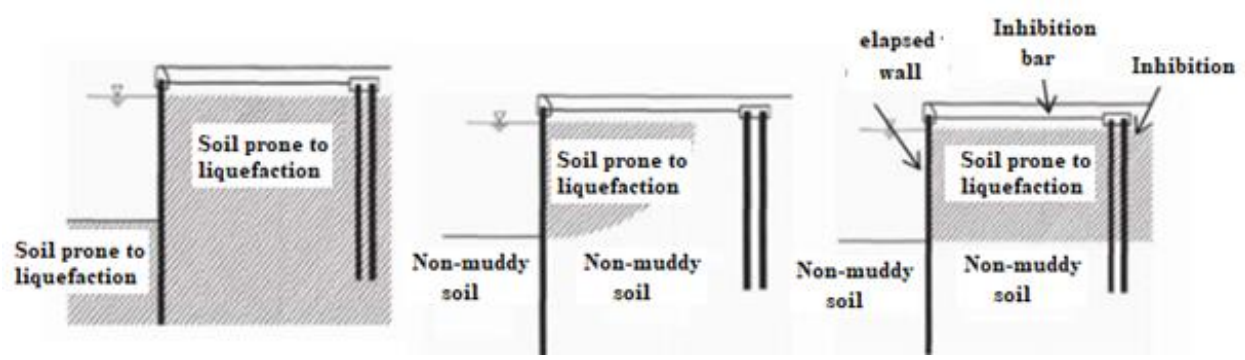

Fig. 1 - Possible States of Rangeland Soil on Site [4]

Table 1 shows the historical data regarding the displacement of the displaced walls of a passage $(d)$ relative to their height from the level of desiccation $(H)$ in the rangeland sites for the earthquakes that are equivalent to the seismicity of the design.

Tab. 1 - Relative Displacement of Septum Walls for Earthquakes Equivalent to Seismic Design Coefficient

\begin{tabular}{|l|l|l|l|l|}
\hline \multirow{2}{*}{ Soil Data } & \multicolumn{4}{c|}{$\begin{array}{l}\text { No Dimensional } \\
\text { Displacement }(\mathrm{d} / \mathrm{H}) \%\end{array}$} \\
\cline { 2 - 5 } & $0-5$ & $5-15$ & $15-25$ & $25-50$ \\
\hline Un-liquefaction & & & & \\
\hline Shell Sand on Surface Only & & & & \\
\hline Shell Sand in Ash and Adjacent Inhibition & & & & \\
\hline Shell Sand on Slopes with Bottom Soil & & & & \\
\hline
\end{tabular}

\section{RESEARCH SIGNIFICANCE}

Due to the limitations of the quasi-static analysis method in the regions susceptible to liquefaction, recent studies in this regard have been primarily focused on the dynamic behavior of shield walls. The main objective of these studies has been to identify the mechanism of breaking the 
walls of the shingles on site. Research in this field has been carried out using laboratory and numerical methods. Laboratory studies have been based on the physical scale simulations of the small scale in the gravimetric field $(1 \mathrm{~g})$ of the seismic table (gravity field) in the form of centrifuge experiments [5-7] and occasionally actual-scale tests [8], while numerical studies have mainly aimed to behave, simulate, and predict these components numerically.

The Japanese NIED Research Institute has conducted experiments using a large seismic table with the size of $3.6 \times 12.6 \times 3$ meters to investigate the seismic behavior of shield walls. These experiments have been modeled using two-dimensional nonlinear dynamical analysis and an effective stress-strain method based on the Tohata-Yay behavioral model. Based on the results the mentioned experiments, the behavior of the set (especially before failure) could be simulated with acceptable accuracy [5]. In addition, the displacement of a damaged shield wall during the 1983 earthquake in the Akita port of Japan was analyzed by Yayy and Kamauka using the same model during the earthquake, and the wall (height: $16 \mathrm{~m}$ ) was displaced 1.1-1.8 meters to the sea due to its soaking liquefaction and cracked at the depth of six meters from the sea level due to the sinking of the steel shield. The analysis also modeled the only behavior before the sinking of the metal wall, and the displacement of the wall crown was estimated with the acceptable accuracy of 1.3 meters [9]. Shuah-Owishi also performed the displacement approximately five meters to the river during the 1964 earthquake due to the soiling and burial section. The numerical modeling of the wall indicated the displacement rate of the horizon by using the mentioned model at 35.3 meters [10]. The obtained results were considered considering the close proximity of the numerical model after the failure of the observation and proper prediction of the variation of water pressure and displacements. Similar studies could contribute to the recognition of the dynamic behavior of shields.

The present study aimed to investigate the effects of the placement of a mating layer on the buried side of an enclosed seabed wall based on laboratory tests and numerical analysis. After introducing the rupture process, the effects of the doping upgrade on the reduction of the shrink displacement were evaluated. The total height of the wall was 35 meters, and the buried length was 14 meters. After the formation of the T-shaped concrete, its placement was restrained by a trickling peg to a candle-buried depth in the soil. Furthermore, sub-surface studies after construction showed that a layer with liquefying potential was part of the wall length of the buried section. For this purpose, a T-model of the failure of a small model of the wall was identified using the seismic table and confirmed for use in various for improvement based on numerical analysis. Dynamic analysis was also performed using the ABAQUS software.

\section{Physical model}

To identify the behavior of the buried seabed walls on a susceptible site to liquefaction, a small sample of the APM Terminals' port wall was constructed based on similarity laws [11] and tested by the seismic equipment. For this purpose, a box composed of transparent Plexiglas was used with the length, width, and height of 180,50 , and 75 centimeters, respectively. The constructive structures (walls, harnesses, and inhibitory cables) were embedded in the middle section of the box, and Plexiglas was used to construct a T-shaped coastal wall and containment. In addition, different layers of soil were poured through silica. The sand had a homogeneous grain size and resembled the standard sandwiches of the Tuyur and Langunaman in terms of the physical properties and granularity curves [12].

To achieve variable degrees of accumulation in the soil layers, we used the wet towering method. For this purpose, $5 \%$ of water was added to the sand, and the soil was meticulously poured into the box in five-centimeter layers to reach the desired percentage. At appropriate stages, the walls, containment, inhibitory cables, and sensors were placed at the predetermined locations. After filling the box, the front side of the wall was partly drilled to apply the pre-stress phase, and after stopping the bar, the front side of the wall was removed to the damp level. To observe the changes in the shape formed during the seismic eruption, a grid of sandstone with the thickness of one and 10 centimeters was prepared to face the box. Upon the completion of the model, it was saturated with 
carbon dioxide gas to remove the air from the open air and accelerate the water saturation process. Model saturation was accomplished by gradually passing the water from the box under constant speed. Figure 2 depicts the geometry of the physical sample. In the non-upgrading model, the loose layer $(\mathrm{Dr}=25 \%)$ contained the entire buried wall surface, while the lower layer and bottom layer had extremely high density $(\mathrm{Dr}=85 \%)$.

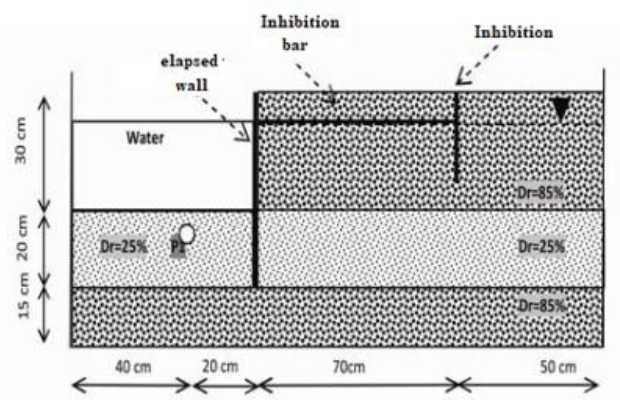

Fig. 2 - Geometry and Layout of Physical Medal without Improvement

An important challenge in this regard is the numerical simulation of a liquefaction phenomenon in which large strains occur in soil and introducing proper behavioral laws for susceptible liquefied soils. Such rules should be able to track the stress near the line of failure during periodic loading, generate a stress-strain loop, calculate the increment of the shear strain, and analyze non-isotropic soil behavior.

One of the models that provides these requirements is based on the multiple shear mechanisms [1], which was introduced by Yai et al. [14, 13] in the FLIP [1] and DIANA software [15]. Dynamic analysis and parametric reference studies [1] have employed this model. Tohata-yay model is a non-linear plastic model, which is based on the large-scale laboratory observation of the behavior of sand in unlade conditions. The main features of the present study are considering the effects of the main stress axis on the effective stress, non-isotropic consolidation, and loading history of soil behavior. The effects of these factors were expressed using two specific concepts known as simple shear virtual mechanism and liquefaction front. Considering the two-dimensional behavior under smooth strain conditions, the effective stress-strain and strain components are as follows [13]:

$$
\begin{aligned}
& \sigma_{z}^{\prime}=\frac{\sigma_{x}^{\prime}+\sigma_{y}^{\prime}}{2} \\
& \varepsilon^{T}=\left(\varepsilon_{x} \cdot \varepsilon_{y} \cdot \gamma_{x y}\right) \\
& \sigma^{\prime T}=\left(\sigma_{x}^{\prime} \cdot \sigma_{y}^{\prime} \cdot \tau_{x y}\right)
\end{aligned}
$$

Considering the fundamental equation of the model, relation (4) divides the mechanism of the rotational motion into two parts. In the first part, the volume-change mechanism is considered, and in the second part (right sentence) the shear modification of the soil is applied without the change of volume [13].

$$
\left\{\begin{array}{c}
\sigma_{x x} \\
\prime \\
\sigma_{y y} \\
\prime \\
\sigma_{x y}
\end{array}\right\}=-B\left(\varepsilon_{P}-\left(\varepsilon_{x x}+\varepsilon_{y y}\right)\right)^{2}\left\{\begin{array}{l}
1 \\
1 \\
1
\end{array}\right\}+\sum_{i=1}^{I} Q_{i}\left(\gamma_{i}\right) \Delta \theta_{i}\left\{\begin{array}{c}
\cos \theta_{i} \\
-\cos \theta_{i} \\
\sin \theta_{i}
\end{array}\right\}
$$

In these relations, $\sigma^{\prime}$ is the effective stress, $\varepsilon$ shows the total strain, and $\varepsilon p$ represents the plastic strain. In addition, coefficient $B$ is as follows:

$$
B=\left(\frac{0.5 k_{m a}}{-\sigma_{m a}^{\prime}{ }^{0.5}}\right)^{2}
$$


where $\sigma_{m a}^{\prime}$ and $k_{m a}$ are respectively the mean effective reference stress and volume modulus of the soil in the predetermined stress. The second sentence of Equation 4 shows the shear deformation of the soil, which was equal to the total shear deformation $(I, i=1, I)$ of the shear mechanism. These onedimensional hypothetical mechanisms or shear modifications are only affected by change, and their vector is considered to be the effect of the main axis of the stress. Scalar $Q_{i}\left(\gamma_{i}\right)$ is defined as the expression $\frac{d Q^{(i)}}{d \gamma^{(i)}}$, which represents the tangential shear modulus relative to, $\Delta \theta$ in the form of $R_{L / U}^{(i)}$, as follows [13]:

$$
\begin{aligned}
& \Delta \theta=\pi / I \\
& R_{L / U}^{(i)}=\frac{d Q^{(i)}}{d \gamma^{(i)}} \Delta \theta
\end{aligned}
$$

In the current research, the volumetric change volume showed the correlations of the effective transformation of the volumetric strains, in which water absorption was considered in addition to the volume change in the soil. The effective stress was also obtained from the tension path calculated by the liquefaction front. Moreover, the lamellar fringes were affected by the same cumulative shear work, which was plotted as a contour in the stress space. Cumulative shear worked up to each step of loading, and the area below the shear strain graph was regarded as the shear stress from the origin of the graph to the step, which partly occurred during the elastic behavior, and the other part occurred during the plastic behavior.

$$
B=\left(\frac{0.5 k_{m a}}{-\sigma_{m a}^{\prime}{ }^{0.5}}\right)^{2}
$$

According to the laboratory observations, the correlations between the cumulative shear work and ductile water overpressure was unique and independent with the stress, shear stress, and axial stress axis of the main stresses up to a certain amount of shear work. In addition, the correlation was transmitted to the tension space without further use, and the concept of the phase change line was applied to divide the tensile space into two expandable and compressible sections. As is shown in Figure 3, a bilinear function of the liquefaction appeared as a variable known as liquefaction frontend $\left(S_{0}\right)$, which is a function of the cumulative shear bonding of the plastic factor without completing each step in the calculation as a function of the modulus soil shear at the medium effective stress level of the same stage.

In summary, the computational process is in the Taha-Yaa model should be described in detail. In each step, the process of transformation, the liquefaction section parameter $S_{0}$ is calculated after calculating the cumulative shear sheathing work. With this parameter and deviation stress ratio, $r$ as the average effective stress ratio of the intermediate model could be determined, and after the multiplication of $S$ to the mean effective initial stress, $\sigma 0 \mathrm{~m}$ volumetric strain plastic $\varepsilon p$ could be obtained from another middle ground. With the calculation of the shear mechanism (4), the effective stresses at the end steps are calculated. Notably, $S_{0}$ shows that the state of liquefaction is the soil and changes over time. $S_{0}=1$ represents the initial conditions (small shear stresses), and $S_{0}=0$ indicates failure due to liquefaction [13].

The parameters required for this model are described in Table 2. In the present study, the specific parameters of the model ( $\mathrm{P} 1, \mathrm{P} 2, \mathrm{~S} 1, \mathrm{C} 1$, and $\mathrm{W} 1)$ were used to calculate the stepwise changes in the soil behavior under the shear load. These parameters were based on trial-and-error and fit the optimal diagram regarding the results of the soil cycling experiments from Qabil three-axis or simple-period cutting $[5,9,10,13,14]$. To determine the parameters, we needed the resistance curve of liquefaction (periodic shear stress as the number of the cycles to reach), $5 \%$ shear strain, and the growth curve of the overpressure of drilling water during the loading cycles. 


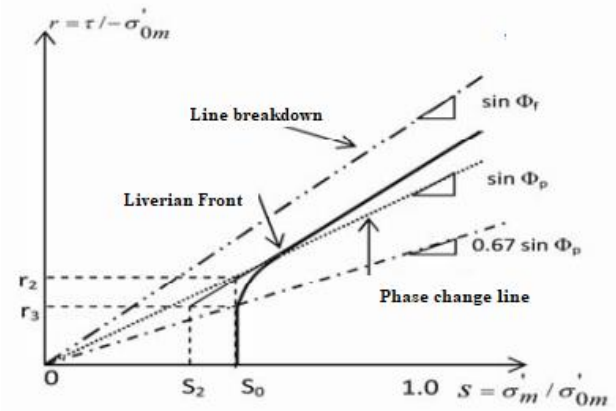

Fig. 3 - Liquefaction Front

Tab. 2 - Introducing Parameters of Tohata-Yay Model

\begin{tabular}{|c|c|c|}
\hline Parameters & Description & Performance \\
\hline$P_{s a t}$ & Effective Medium Reference of Tension & - \\
\hline$P_{r e f}$ & Volumetric Modulus Change Shape & Volumetric \\
\hline$K_{m a}$ & Modulus Shear & Shear \\
\hline$G_{m a}$ & Internal Angle Friction & Shear \\
\hline$\varphi_{f}$ & Angle Phase Change & Dilation \\
\hline$\varphi_{p}$ & Control Effect of Cumulative Shearing Work & Dilation \\
\hline $\mathrm{W} 1$ & Threshold of Shearing Work & Dilation \\
\hline $\mathrm{C} 1$ & $\begin{array}{c}\text { Control of First-phase Increase in Pressure } \\
\text { Hole }\end{array}$ & Dilation \\
\hline $\mathrm{P} 1$ & $\begin{array}{c}\text { Hole } \\
\text { Control of Second }\end{array}$ & Dilation \\
\hline $\mathrm{P} 2$ & Control of Lower-limit of Front Liquefaction & Dilation \\
\hline $\mathrm{S} 1$ & Hysteric Coefficient & Shear \\
\hline $\mathrm{Hv}$ & &
\end{tabular}

After modeling the conditions of the soil samples in periodic testing using software, the following steps were performed, and errors occurred in estimating the model parameters.

- For the initial estimation and prevention of the infinity strains $\left(S_{1}\right)$, take a small positive amount of about 0.005 until $S_{0}$ does not exactly equal zero.

- The normal shear rate is in the lower values, and the water supply produces no pressure. As the initial guess, $C_{1}$ is taken to be one since this parameter is effective. However, no significant increase is observed in the strain (P1, W1, and P2) for the large strains that are determined by the initial guess $(\mathrm{C} 1=1)$.

- W1 and P1 were determined by comparing the analytical hole pressure overpressure curve and laboratory based on section $r_{u}<0.6$. Since $\mathrm{W} 1$ does not dominate $\mathrm{P} 1, \mathrm{~W} 1$ starts with a good assumption and is determined from $\mathrm{P} 1$. The prime premise for $\mathrm{P} 1$ is a value within the range of $0.4-$ 0.7 , and after determining $\mathrm{P} 1, \mathrm{~W} 1$ is precise. The larger $\mathrm{W} 1$ and $\mathrm{P} 1$ show the slower growth of water pressure. Notably, the ratio of the excess water pressure to hole $r_{u}=\frac{\Delta u}{\sigma_{v 0}}$ in which the hole pressure is added with $\Delta u$ and the effective initial verbal stress, $\sigma_{v 0}$ is shown by comparing the growth curves of the excess water pressure of the cavity calculated, while based on section, $r_{u}>0.6$ is estimated. In the case of calculating the shape deformation, the soil and pew structures are required, and this parameter is more acceptable by comparing the computational and experimental curves of the shear strain that has been estimated by the loading cycle. In this regard, the higher P2 indicates that the increased slice strain amplitude is faster. After estimating the mentioned parameters for the large strains, their suitability is assessed in the small strains. With a model for software analysis, these 
values are not considered suitable for the laboratory values of the lower strains, and the $\mathrm{C} 1$ changes [14] are higher for the loose soils (C1) [16].

\section{NUMERICAL SIMULATION}

To maintain the correlation of the numerical model with the actual project, the geometric properties of the sections were considered similar to Shahid Rajaee port coastal wall. Figure 4 illustrates the general scheme of the numerical models in the current research. In the selected areas, four sections were observed with a backfill as the first layer, and the second layer was divided. In the first layer, two areas were adjacent to the burial side of the bumper, which were the region behind the root and the front of the root. In all the analyses, the landing and second layer were modeled with dense aggregates, and the first sub-sets varied. Therefore, the naming of the models was due to the material disparity carried around the buried section, and the model without refurbishing a set and no front behind the root of the wall was not condensed.

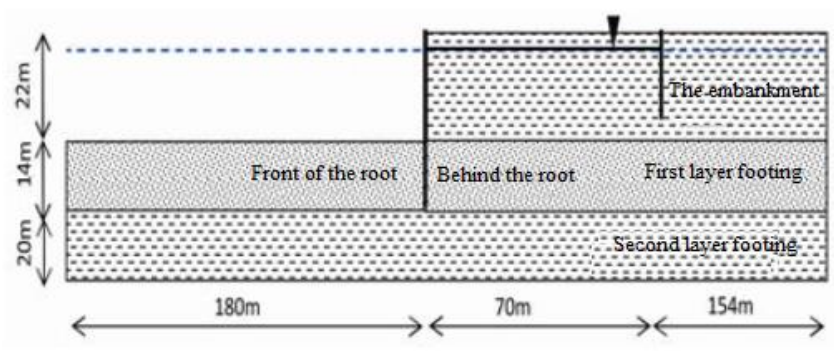

Fig. 4 - Public Plan of Numerical Models

The main constituent of the limited components of this model has the size of $404 \times 56$ meters, and the meter is located in the central part of the building. Soil compositions with the eightcomponent tetrahedral elements of strain flat, anchor bar with two-point springs, shields, and harnesses unbound the shell elements and could be networked [15]. To prevent numerical problems, dimensional elements are required depending on the input wavelength to the environment [17], and the dimensions of the largest element in terms of the base acceleration frequency and wavelength could be selected. The weakest material is selected to obtain more accurate responses, and the elements in the backfill and in the vicinity of the shield to the front smaller restraint are also considered. Due to the executive process, construction of the exterior walls, and calculation of the initial stresses, a step-by-step analysis method was used in the current research. Initially, the flat ground was analyzed under the load weight, followed by the activation of the shield and shield elements, and the set was balanced. At the third stage, the front side of the wall was removed to the barrier level after establishing the balance condition, the bar was activated as well.

In the fourth step, the bar was applied by an axial force equivalent to the flattened strain condition to its end node. By defining a similar force in the opposite direction to the corresponding node of the bar on the shield and the 'matched motion' condition for the two knots, an obstacle rod was attached to the wall during the fifth stage and the front side of the wall, reaching the dripping line of -22 meters. These stages were analyzed in the static state. The static analysis was performed using Moher-Kolumb model, and drainage conditions and tension were also employed.

At the fifth stage, the initial tension was transferred to the dynamic analysis stage. At the outset of the dynamic analysis stage, there was no drainage, and the behavioral model was changed into the Tohata-yay model. In total, 10 seconds of ground motion acceleration was performed at twosecond steps for the seismic analysis of the samples. The maximum and minimum automatic time of the calculations was one and 0.0001 seconds, respectively, and these stages were performed by controlling the integral time error. The dependence of the controller was obtained as the time step, and in the case of dependency, the time step was corrected. 
The Runge-Kutta time-integral method consists of two steps. With integration in every step of the procedure, the Newton-Raphson and convergence criterion as a ratio increase the unbalanced force selected for the external forces. To assess the effects of frequency and base acceleration in the present study, the models were accelerated with the maximum range of 0.25 and $0.50 \mathrm{gram}$. Per acceleration of the harmonic input, the frequencies of 1,2 , and $3 \mathrm{~Hz}$ were applied. These specifications were selected so that in addition to the occurrence of liquefaction in a set without improvement, the effect of continuity could be determined by using the rotational load before and after leakage [17]. Therefore, the dimensions of the largest element were selected based on the base acceleration frequency and wavelength in the weakest materials.

Smaller containment responses were considered, and to implement the construction of the shield walls and calculate the initial stresses, the step-by-step analysis method was used. For this purpose, flat ground was initially analyzed under the load weight, and with the activation of the shield and control elements, the set was more accurately balanced. In addition, the elements in the region of the earth and vicinity of the shield to the front in the third stage and the front wall were removed to the level of the barrier. After activating the balance condition, the barrier element was also activated. In the fourth step, the bar was subjected to an axial force equal to the strain condition of the flat end its end node. By defining a similar force in the opposite direction to the corresponding node, an obstacle rod on the shield and a 'matched motion' condition for the two knots were attached to the wall with an obstacle rod.

At the fifth stage, the soil from the front of the wall that reached the line $(22 \mathrm{~m})$ was removed, and the analysis of the steps was static. The static analysis of the Mohar-Coulomb model and drainage conditions were also performed, and the tensions and initial stresses of the fifth stage were transferred to the dynamic analysis stage. At the outset of the dynamic analysis stage, the conditions were not drained, and the behavioral model was changed into the Tohata model. The base acceleration was applied for 10 seconds in steps of 0.01 second. The automatic time steps were determined with the maximum of one second and minimum of 0.0001 second using the ground fault controller, and the integration was performed. The dependency controller examined the response as far as the time step, and corrected the time step in the case of dependency.

To control the two steps, the Runge-Kutta integral method was used in this method. Integration in each step of the time was performed by the Newton-Raphson method, and the convergence criterion was selected as increased ratio of the unbalanced force to the external force [18]. To investigate the effects of frequency and base acceleration, the models were exposed to base acceleration with the maximum range of $0.25-0.50 \mathrm{gram}$. In the acceleration steps, the harmonic input moved at the frequencies of 1,2 , and $3 \mathrm{~Hz}$. These specifications were selected so that in addition to the occurrence of liquefaction in an unsorted set, the effect of the continuity of the load application would be assessed before and after liquefaction.

Tab. 3 - Specimen Properties

\begin{tabular}{|l|l|l|l|l|l|}
\hline Member & Section & $\mathrm{P}\left(\mathrm{kg} / \mathrm{m}^{3}\right)$ & Dimension & Ball length & Thickness \\
\hline Wall & T-shape & 2500 & $4^{\star} 4$ & 4 & 0.8 \\
\hline Inhibition & Circular & 2500 & 1 & - & - \\
\hline
\end{tabular}




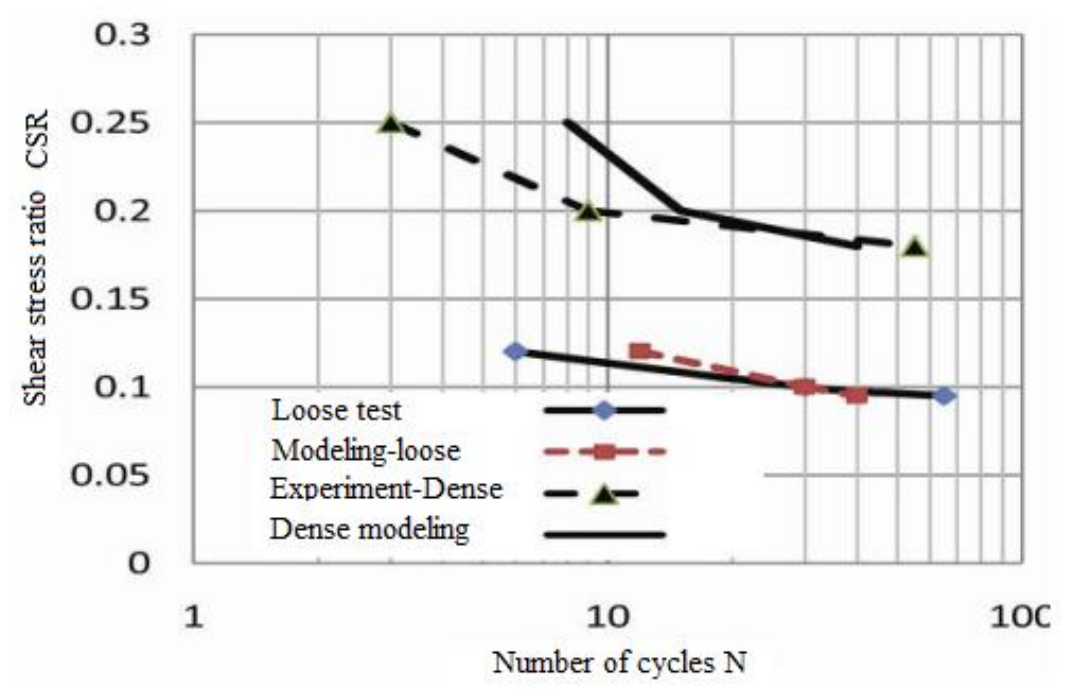

Fig. 5 - Modeling of Sand Resistance against Liquefaction

Tab. 3 - Characteristics of Soil Materials

\begin{tabular}{|l|l|l|l|l|l|l|l|l|l|l|l|}
\hline Situation & $P_{s a t}\left(\mathrm{~kg} / \mathrm{m}^{3}\right)$ & $K_{m a} \mathrm{MPa}$ & $G_{m a} \mathrm{MPa}$ & $\varphi_{f}$ & $\varphi_{p}$ & $\mathrm{~W} 1$ & $\mathrm{C} 1$ & $\mathrm{P} 1$ & $\mathrm{P} 2$ & $\mathrm{~S} 1$ & $\mathrm{Hv}$ \\
\hline Weak & 1900 & 223 & 86 & 34 & 28 & 4 & 1.5 & 0.3 & 3.8 & 0.005 & 0.24 \\
\hline Dense & 1980 & 300 & 115 & 40 & 28 & 40 & 1 & 0.1 & 1.0 & 0.005 & 0.24 \\
\hline Very Dense & 2000 & 324 & 125 & 42 & 28 & 42 & 1 & 0.1 & 0.8 & 0.005 & 0.24 \\
\hline
\end{tabular}

To determine the soil parameters in loose and dense compaction conditions for use in the Taha-Yaah model, cyclic experiments were performed on the sand specimens with the density of $35 \%$ and $75 \%$ [19]. After the numerical modeling, the specimen conditions were determined in the cyclic and by the simultaneous comparison of the percolation water pressure and shear strain with the measured values using the Taha-yea model parameters in the form of trial-and-error. Given the importance of shear strain over excess water pressure, the lubrication criterion reached the strain amplitude of $\mathrm{DA}=5 \%$ in determining the parameters. In addition, the soil areas were modeled with extremely dense soil parameters (95\%). Figure 5 shows the comparison of the results of the numerical model in these samples with the experimental values. The parameters used for the analysis are presented in Table 4. Considering the similarity of the selected mass to the standard Taureau [12], the maximum shear modulus and GMA of the proposed correlations with the Taoyu sand were also determined [20].

\section{RESULTS AND DISCUSSION}

In the current research, the unshaped physical model was located at $0.25 \mathrm{gram}$ and the frequency of $3 \mathrm{~Hz}$ after saturation and subjected to sinusoidal acceleration. Figures 6-a and 6-b respectively depict the initial and final state of the unmodified model and SPM1. By applying the base acceleration, the foot of the wall that was immersed in the soil susceptible to liquefaction was displaced more than the crown of the wall. This type of failure is referred to as 'burst section failure'. Due to the wide affected areas, including all the components of the buffer structure, the repair of the structure after the occurrence of this mode might be very costly and even impossible.

According to section $\mathrm{B}$, the path of the color lines indicated that most of the deformations were concentrated in the loose soil near the root of the wall. In addition to the significant elevation of the bottom of the sea, the movement of the sidewall also significantly reduced the backing effect of this layer to the buried wall section, thereby leading to the large period, followed by an active wedge from the buried side to the back of the containment. Due to the fact that the barrels were located in this wedge and the movement alongside, the braces also suffered considerable periods. 
The shaped areas in front of the root and the sea, along with the horizon, compressed and extended along the vertical axis, while behind the root, the deformation portion was compressed along the horizon and extended along the vertex. With this type of deformation on both sides of the root, a combination of flattening and cutting would be possible with different marks. Due to its low weight and low wall density, no significant changes occurred in the soil under the root in the current research. On the other hand, the embankment summit increased to the wall and decreased in depth, which could be attributed to density, the lateral movement of the wedge, and the combination of these factors.

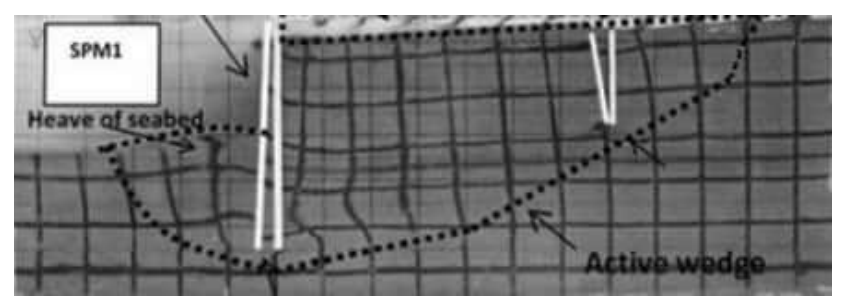

(a)

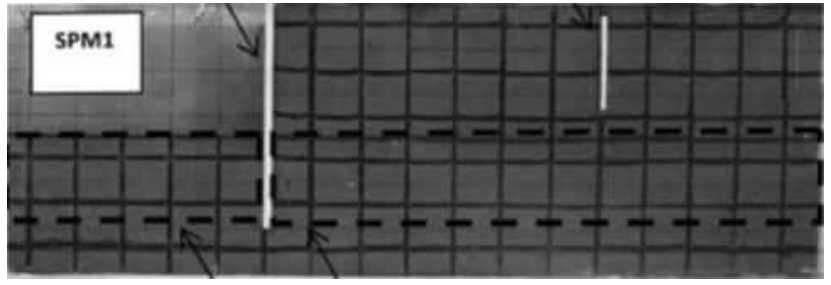

(b)

Fig. 6 - a) Original Form, b) Final Shape of Ungraded Model

As can be seen in Figure 7, the wall crown shifted to the sea during a shake of 97.4 centimeters, which was equivalent to the wall height of $16 \%$. Given the final shape of the model, the displacement of the foot of the wall was clearly greater than this ratio. As is shown in Figure 8 , the displacement occurred simultaneously with the significant increase in the ratio of the excess water pressure and the soil liquefaction in the front of the root of the wall at the distance of 20 centimteres (Figure 2) (points P1).

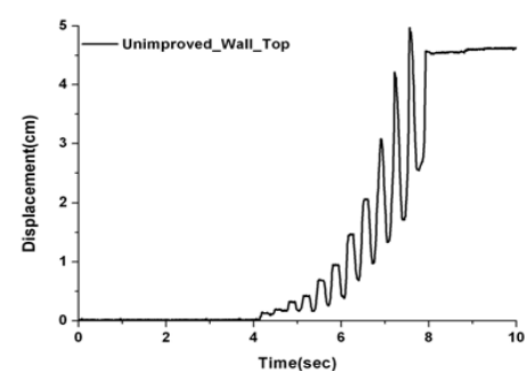

Fig. 7-Movement History of Crown of Wall

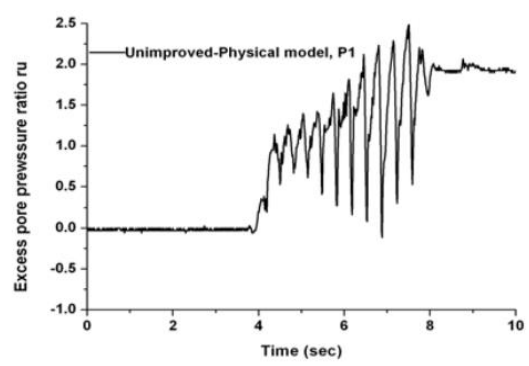

Fig. 8 - History of Water Drainage Ratio Simulation

The introduced set in the numerical model underwent significant changes after applying the base acceleration. Regardless of the amount of these deformations, which depended on the features of the base motion, the overall geometry of the set was similar to (9), indicating the reversal of the restraint and the wall toward the sea. In addition to lateral movement, a considerable amount of seating was observed at the crown and foot of the wall.

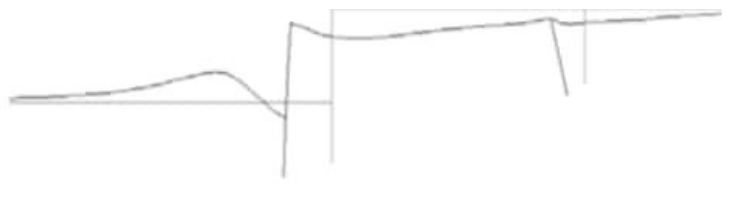

(a)

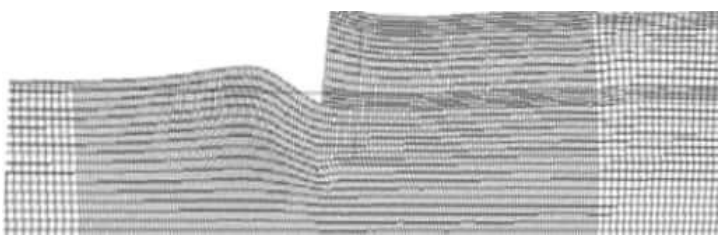

(b)

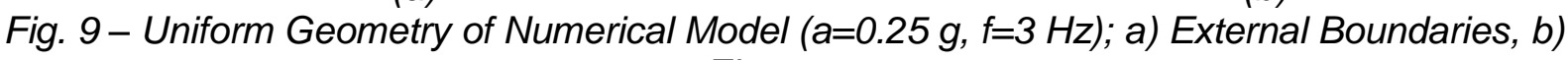
Elements 
In the current research, soil sedimentation, its lateral displacement, and the elevation of the bottom of the sea were also considered as the most important changes in the soil layers. According to section $\mathrm{B}$, most of the deformations were concentrated in a clear range, which started from the bottom of the wall and extended along one or two straight lines to the back of the containment. The other part was the level of the rupture in front of the root of the wall, and the sea was formed on the side. In contrast to the shape deformation of the structure, the deformation of the other parts of the complex was negligible. Furthermore, the final geometry of the collection was observed to be close to the laboratory sample. Therefore, it could be concluded that assessing the effectiveness of the upgrading soil around the root is of particular importance. Figure 10 indicates that the displacement slope of the foot of the bot was higher than the top of the tower due to the fact that the foot of the wall was loose in the loop, thereby causing the change of the failure mode to 'bursting section failure' based on the acceleration period during which the change of the foot of the wall became larger than the crown. In addition, the increased frequency of the input motion caused the displacement values to significantly decrease, and the change in the failure mode occurred later on. For instance, while displacing the foot of the wall during the inertia movement with the frequency of $1 \mathrm{~Hz}$ at the angle of 4.8 seconds from the crown displacement, it occurred during the base acceleration, which was similar to the frequency of $3 \mathrm{~Hz}$ at 6.8 seconds.

The final displacement of the wall crown was observed at the frequency of $4 / 00,1 \mathrm{~Hz} \mathrm{~m}$ $(18 \%)$, and the height of the wall of the dripping code (frequency: 62.2 and $2 \mathrm{~Hz}$ ) was estimated at $12 \%$. In addition, the wall height of the dripping code (frequency: 14.2 and $3 \mathrm{~Hz} \mathrm{~m}$ ) was $9.72 \%$. as is shown in Figures 10 and 11, the history of the change of wall location with the increased acceleration of the system caused the wall foot fracture to become faster, and a larger wall than the accelerator had larger displacements at all the moments. For instance, the final displacement of the wall crown during the acceleration of 0.50 gram and frequency of $20.51 \mathrm{~Hz} \mathrm{~m}(23 \%)$ of the wall height of the dripping code (frequency: 97.3 and $2 \mathrm{~Hz} \mathrm{~m}$ ), $18 \%$ of the wall height of the dripping code (frequency: 19.3 and $3 \mathrm{~Hz} \mathrm{~m}$ ) was estimated at $14 \%$. On the other hand, the height of the wall was of a desiccant code, and the doubled base acceleration rate could not increase the displacement doubles.

As is depicted in Figure 12, the endless displacement of the bezel of the wall crown decreased in terms of height due to the simulation in the studied accelerations, with an almost equal slope due to the higher frequency. Figure 13 shows the strong dependence of the displacement on the input frequency, the importance of this parameter in the analysis of the seismic behavior of the set, and the results of the wall arrangement. Correspondingly, a significant meeting occurred under the wall during the baseline vibration with the accelerated velocities, and this arrangement increased with higher acceleration, while decreasing at the higher frequency of the inertial movement.

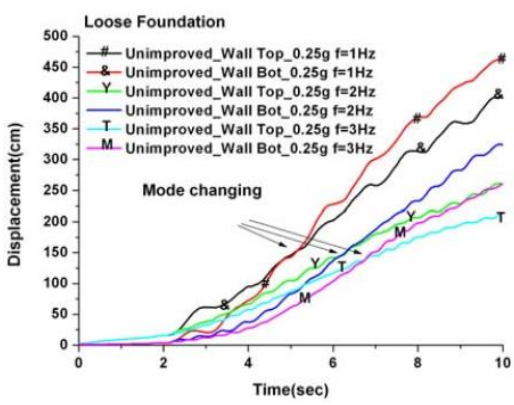

Fig. 10 - Comparison of Failure of Wall in Ungraded Site during Base Acceleration of $0.25 \mathrm{~g}$ and Frequency of 1 , 2, and $3 \mathrm{~Hz}$

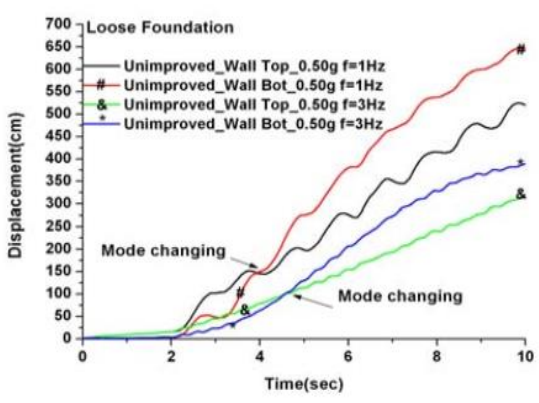

Fig. 11 - Comparison of Failure of Wall in Ungraded Site during Base Acceleration of $0.25 \mathrm{~g}$ and Frequency of 1 , 2, and $3 \mathrm{~Hz}$ 


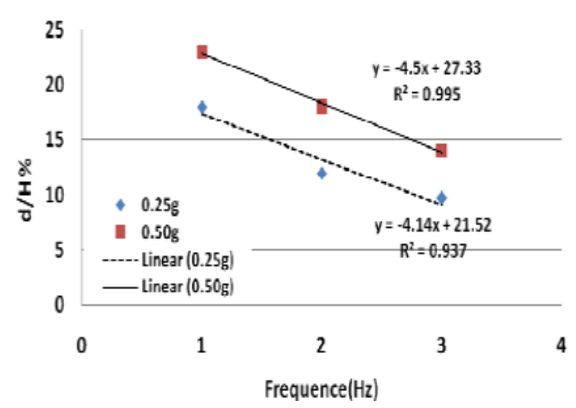

Fig. 12 - Dependence of Displacement between Crown Dimension of Wall and Input Frequency in Unshared Site

According to the findings of the current research, the loose-layer liquefaction was denser compared to the layers that were prone to increased water pressure, which could lead to less leakage in the areas with lower primary stress. Figure 16 shows the position of several elements examined in the loose layer. The horizontal distance of the P2, P1, and P3 elements from the root of the wall was estimated at 20,10, and 30 meters, respectively, and the horizontal distance of the P4 components was 10 meters. Furthermore, the elements examined from the second element of the loose layers had higher initial tensile stress compared to the upper elements without a common knot with the elements in the dense aggregates.

As is depicted in Figure 14, the monotonic component of the cavity water pressure ratio (run) in the front and near the root of the wall grew more significantly than the particle elements, while this ratio decreased significantly with the increased distance of the element from the root of the wall at a considerable rate. This is because the elements were close to the fringe and had static shear stress, which increased the shear strain and liquefaction potential of the soil [17]. The presence of smaller effective overhead and initial shear stress also caused these areas to behave as the weakest part of the complex in the event of soil loosening and accelerate in the strongholds.

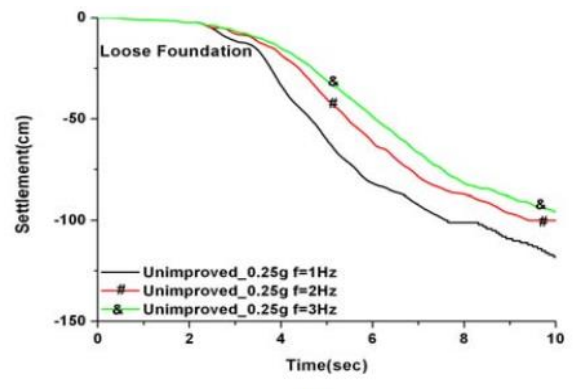

(a)

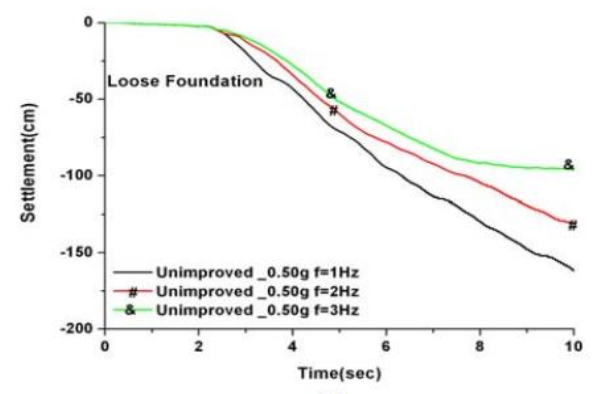

(b)

Fig. 13 - Seating of Wall in Unmodified Site at Frequencies of 1, 2, and $3 \mathrm{~Hz}$ with Base Acceleration of a) $0.25 \mathrm{~g}$ and b) $0.50 \mathrm{~g}$

Based on this form, while the elements were located at 10 meters in front of the wall during the acceleration of $0.25 \mathrm{gram}$ and liquefied, the soil was located at 30 meters (approximately twice the depth of the buried section), tolerating a small load of $25 \%$ of the initial stress. It was also observed during this loading in the soil, the elements were loose behind the wall due to the larger overhead, and the excess water pressure could not be produced, thereby leading to no risk of liquefaction in these areas. 

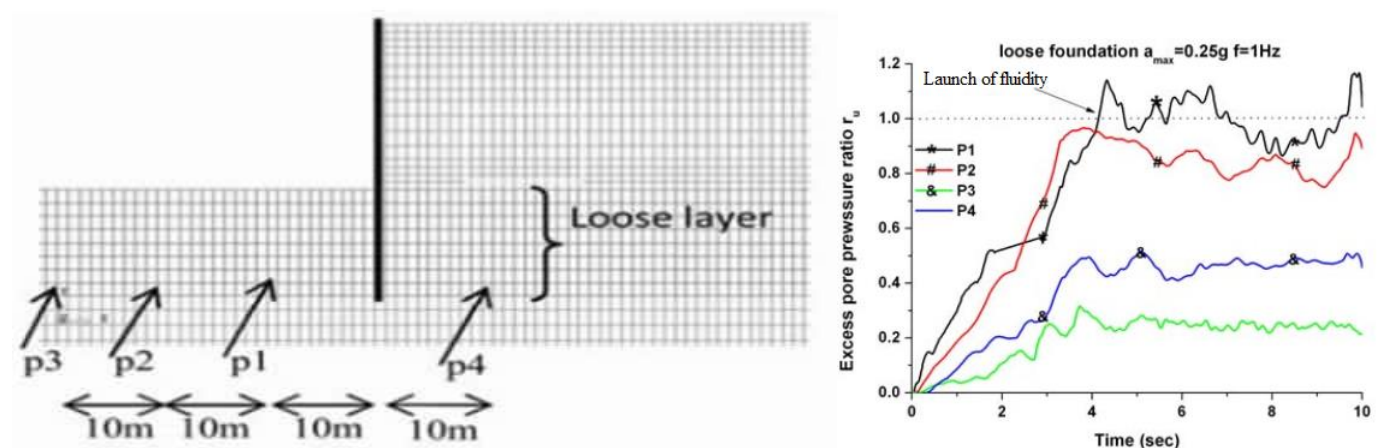

Fig. 14 - Liquefaction of Front and Back Elements of Wall with Base Acceleration of $0.25 \mathrm{~g}$ and Frequency of $1 \mathrm{H}$

Considering the design of the shield walls based on the assumption of the joint foot or groove operation, the monotonic component of the flexural anchor of the foot of the wall was extracted in order to recognize the changes in the ankle of the wall in this situation. During the dynamic loading, if the anchor was applied to the wall contrary to the anchor's direction, the median part of the free wall length could be obtained. Therefore, it could be concluded that the soil around the root of the wall acts as a support and behaves at the foot of the wall. Figure 15 shows the comparison of the curvature of the bending anchor curves of the wall at $1,0,0.1$, and 5 seconds, gravity of 0.25 gram, and frequency of $1 \mathrm{~Hz}$. It was also observed that at the outset of the loading, an anchor resistant to the buried section (distance from the wall foot: 14-0.0 m) was in the opposite direction to the anchor, affecting the mid-section of the free-wall length. Over time, not only the anchor was destroyed, but it extended the free length into the buried section. The time history of the monotonic component of the bending anchor of the foot of the wall (Figure 16) indicates that the growth of the anchor began from the initial moments of the base acceleration due to the lack of a change in the monotonic anchorage entering the middle section of the wall at different accelerations in terms of the signs. The presence of this anchor with an anchor on the wall and a loose layer around the buried section not only produced a rigid anchor in this section, but another anchor entered the middle section with a stimulus state and unstable in the wall.

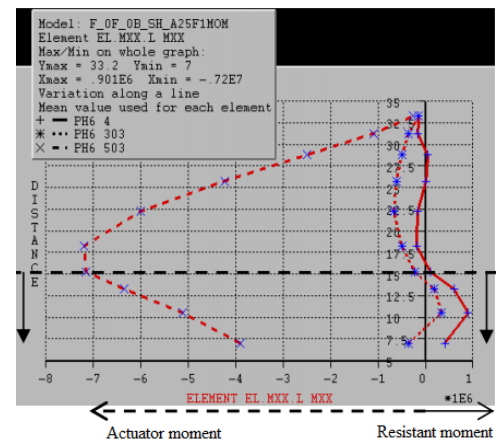

Fig. 15 - Variation of Bending Anchor of Wall at Reconstruction Site (second PH6 503) (5) and PH3 (303) 3, (PH6 4) 0/01 Not Found during Base Acceleration of $0.25 \mathrm{~g}$ and $1 \mathrm{H}$ Frequency
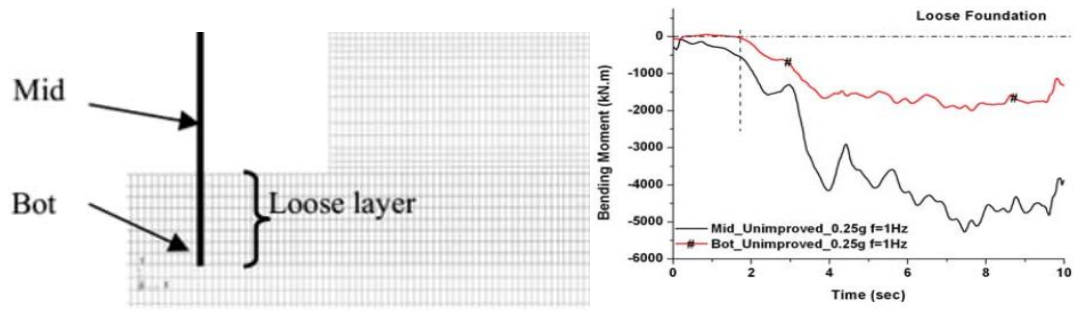

Fig. 16 - History of Bending Anchor in Middle and Foot of Wall in Non-Upgraded Site 
The presence of an anchor actuator at the foot of the wall suggested that even assuming a joint foot in the susceptible site to liquefaction would be hypothesized to be a loss if the entire buried section was in such a layer.

\section{IMPROVEMENT AROUND THE ROOT}

As mentioned earlier, density was used as the method of improvement in the present study. In this section, the effects of the bulk density of the sides of the section have been described with an emphasis on the wall displacement and bending anchor variations. The basis for selecting the front side of the wall was to improve the liquefaction, which started from the soil of this area. It seemed that the improvement of this section would reduce the displacement of the set or change the failure mode. Due to the fact that the lateral pressure of the loose soil was denser than the soil, it seemed that the improvement of the soil behind the wall would reduce the pressure on the wall given the importance of identifying the effects of the improvement length on the set performance. Furthermore, the behavior of the models in the I-2.5-B-2.5 and I-5-B-5 designs were evaluated at $1 \mathrm{~Hz}$, where I and B represented the equivalent soil height in the front and behind of the wall (Figure 19).

In these designs, the front and back sides of the wall were about five and 12 times the length of the buried wall with very dense material. The improvement of the two sides of the roots in the studied lengths significantly decreased the displacements, and Figure 17 shows that during the base acceleration of 0.5 gram and $1 \mathrm{~Hz}$ frequency in the model I-2.5-B-2.5,the displacement of the wall crown decreases from $25 \%$ of the wall height relative to the dredging level to $18 \%$ and the displacement of the wall foot from $30 \%$ to $15 \%$. The design also prevented the foot of the wall from the wall crown, making it easier to repair the operations in the absence of shield bomber damage.

Figure 18 shows a posture without the crown and foot of the wall compared to the height of the dairy code at the base acceleration of 0.25 and 0.5 gram. With the execution of the I-2.5-B-2.5 design in both moves, the footprint of the foot of the wall was corrected from its crown. By the fivefold increase of the repair length on each side, the displacement of the crown and foot of the wall decreased more effectively. Due to the small difference in the results of the I-5.0-B-5.0 model, the optimal optimization time for the provided specification was less than five times the buried depth on the sides of the root.

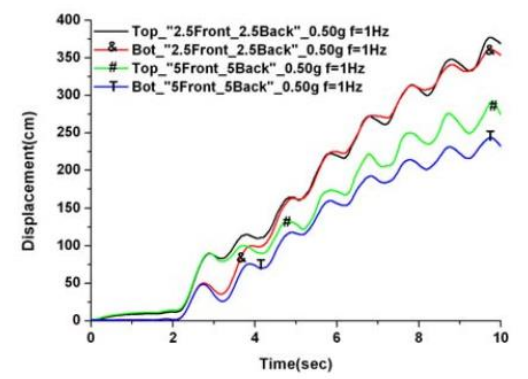

Fig. 17- History of Displacement of Peak and Foot of Wall in Models I-2.5-B-2.5 and I-5.0-

$B-5.0$ at Acceleration of 0.5 and $1 \mathrm{~g}$

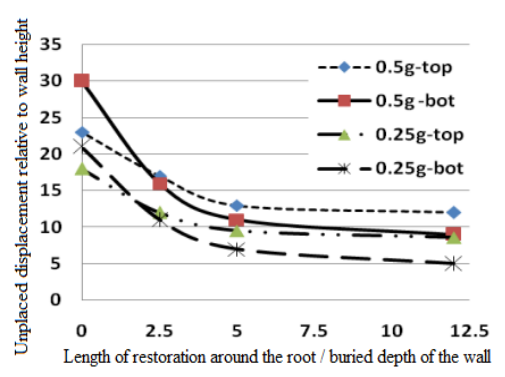

Fig. 18 - Effect of Length of Recovery on Displacement

To justify the effects of the proposed designs, attention should be paid to the changes in the bending anchor on the middle section and foot of the wall. For this purpose, in the form (19), the variation of the monotonic curvature anchor was denoted in the middle and foot of the wall at the acceleration of $0.5 \mathrm{~g}$ and $1 \mathrm{~Hz}$ frequency for the unmodified model. Unlike the ungraded model in which there were the joint foot conditions with an anchor zero at the foot of the wall (only lasted two seconds), improvement was observed in the sides and maintained the behavior to the end of the vibration. In addition, median wall anchor significantly decreased, and the same trend was observed in the other improvement projects. 


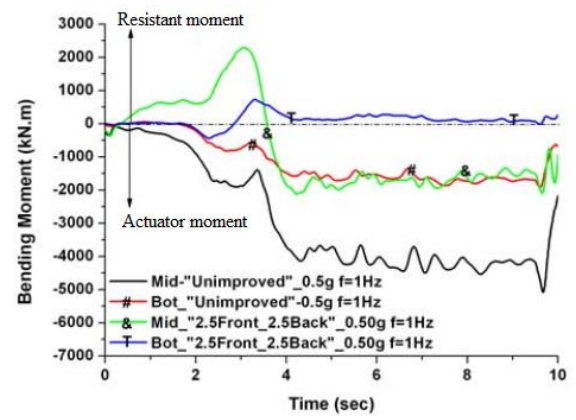

Fig. 19 - Wall Bending Anchorage Model I-2.5-B-2.5 at Acceleration of $0.5 \mathrm{~g}$ and $1 \mathrm{~Hz}$ Frequency

\section{CONCLUSION}

In the current research, the effects of a lubricant susceptible layer around the buried section of an enclosed seabed wall using physical and numerical modeling were investigated in terms of the base acceleration and intake frequency, and the efficiency of the back and front portions of the buried section in the reduction of the wall displacement and variation was also assessed. Furthermore, the failure mode was evaluated, and the obtained results indicated that if there is susceptible layer liquefaction around the root of the wall, the front of the root on the seafloor increases the cavity water pressure excess ratio due to the lower initial effective stress and presence of the initial static shear stress. Leakage in the front of the root and the large lateral pressure on the root of the root also significantly increased the likelihood of the bursting fracture failure, and the failure could primarily be attributed to the formation of the soil recessive effect around the buried section, as well as the use of an anchor actuator in this section.

According to the results, the dependency of the sharp change of the locations on the base acceleration and frequency of the entrance motion highlighted the importance of these factors in the seismic behavior of the set. As a result, the application of a larger base acceleration led to large displacements, while the displacement increased at the higher frequencies of the inertia movement. By improving the soil on both sides of the root and preventing the bursting fracture, the displacement of the crown and foot of the wall significantly decreased. In addition, the effects of improving the sides of the root on the reduction of the displacement of the foot of the wall were considered more significant compared to the reduced displacement of the crown of the wall. Finally, the minimum required length of the root grooves for the studied designs was observed to be 2.5 times the length of the root of the wall to prevent the failure of the buried section, while the reverse process did not show a significant increase after the five-fold upgrade to the root length.

\section{REFERENCES}

[1] International Navigation Association, "Seismic Design Guidelines for Port Structures", Balkema, Tokyo, 2001.

[2] Ramos, M.C. \& Mart'nez-Casasnovas, J.A., " The cost of soil erosion in vineyard fields in the PenedesAnoia Region (NE Spain)", Catena 68194 - 199. 2006.

[3] Psarropoulos, P. N., Klonaris, G., Gazetas, G., "Seismic Earth Pressures on Rigid and Flexible Retaining Walls", Soil Dynamics and Earthquake Engineering, 2005, 25, 795- 809. [4] Mondal, G.C. \& Rai, D. (2008), " Performance of harbor structures in the Andaman Islands during the 2004 Sumatra earthquake", Engineering Structures 30 174-182.

[5] Mohajeri, M., Kobayshi, Y., Kawaguchi, K., Sato, M., "Numerical Study on Lateral Spreading of Liquefied Ground Behind a Sheet Pile Model in Large-Scale Shake Table Test, " The 13th World Conference on Earthquake Engineering, Vancouver, Canada, 1-6 August 2004.

[6] Motamed, R., Towhata, I. "Shaking Table Model Tests on Pile Groups Behind Quay Walls Subjected to Lateral Spreading", Geotechnical and Geoenvironmental Engineering, 2010, 136, 477-489.

[7] Ueda, K. Tobita, T., Iai, S. "A Numerical Study of Dynamic Behaviour of a self-supported Sheet Pile Wall", The 14th World Conference on Earthquake Engineering, Beijing, China, 12-17 October 2008. 
[8] Kohama, E., Sugano, T, Shiozaki, Y., Mutoh, M., "Dynamic Behaviour of a Steel Sheet Pile Quay Wall in the Full-Scale Experiment with Regard to Liquefaction", The 13th World Conference on Earthquake Engineering, Vancouver, Canada, 1-6 August 2004.

[9] Nozu, A., Ichii, K., Sugano, T., "Seismic Design of Port Structures", Journal of Japan Association for Earthquake Engineering, 2004, 4 (3).

[10] Sawada, S., Ozutsumi, O., lai, S., "Analysis of Liquefaction-Induced Residual Deformation for Two Types of Quay Walls: Analysis by FLIP", The 12th World Conference on Earthquake Engineering, Auckland, New Zealand, 30 January-4 February 2000.

[11] lai, S., "Similitude for Shaking Table Test on Soil-Structure-Fluid Model in 1g Gravitational Field", The Port \& Harbour Research Institute, Japan, 1988.

[12] Ghalandarzadeh, A., Akbari-Paydar, N., "Shaking Table Tests of Flexible Quay Walls Subjected to Backfill Liquefaction", Asian Regional Conference on Soil Mechanics and Geotechnical Engineering, New Delhi, India, 2007, pp 690-693.

[13] I ai S., Matsunaga, Y., Kameoka, T., "Strain Space Plasticity Model for Cyclic Mobility", Report of the Port and Harbour Research Institute of Japan, 1990.

[14] Iai, S., Matsunaga, Y., Kameoka, T., "Parameter Identification for a Cyclic Mobility Model", Report of The Port and Harbour Research Institute of Japan, 1990.

[15] Manie, J., Kikstra, W. P., "DIANA User's Manual: Matlab", 1st Edition, TNO DIANA BV, Netherlands, 2009, pp 475-479.

[16] I chii K., S. lai, Y. Sato H. Liu, "Seismic Performance Evaluation Chart for Gravity Type Quay Walls", Journal of Structural Engineering / Earthquake Engineering, JSCE, 2002, 19, 21-31.

[17] Kramer, S. L., "Geotechnical Earthquake Engineering", Prentice Hall, 1996.

[18] Manie, J., Kikstra, W. P., " DIANA User's Manual: Analysis", 1st Edition., TNO DIANA BV, Netherlands, 2009, pp 497-499.

[19] A hmadi, M., Shirasb, A.," Liquefaction Resistance of Firozpur Sand using Cyclic Simple Shear Test", The 6th National Conference on Civil Engineering, Semnan, Iran, 26-27 April 2011.

[20] Arable, A., Ghalandarzadeh, A., Mostafagharabaghi, A. R., Abedi, K., "A Numerical Study of Liquefaction-Induced Deformation on Caisson-Type Quay Wall Using a Partially Coupled Solution", Journal of Offshore Mechanics and Arctic Engineering, 2010, 133 (2). 